\title{
Excitation transfer in C-phycocyanin. \\ Förster transfer rate and exciton calculations based on new crystal structure data for C-phycocyanins from Agmenellum quadruplicatum and Mastigocladus laminosus

\author{
Kenneth Sauer ${ }^{a}$ and Hugo Scheer ${ }^{b}$ \\ ${ }^{a}$ Chemistry Department and Chemical Biodynamics Laboratory, Lawrence Berkeley Laboratory, University of California, \\ Berkeley, CA (U.S.A.) and ${ }^{b}$ Botanisches Institut der Universitär München, München (F.R.G.)
}

(Received 29 February 1988)

Key words: Photosynthesis; Antenna; Energy transfer, picosecond kinetics; Phycocyanin; Förster mechanism: Exciton

Calculations of excitation transfer rates among the chrialophores of C-phycocyanin using the Förster inductive resonance transfer mechanism have been carried out using the new coordinates for the position and orientation of the chromophores (Schirmer, T., Bode, W. and Huber, R. (1987) J. Mol. Biol. 196, 677-695). Several of the rate constants are significantly altered from the results of our calculations using the previously published coordinates (Sauer, K., Scheer, H. and Sauer, P. (1987) Photochem. Photobiol. 46, 427-440). In particular, for the $(\alpha \beta)_{3}$-trimers of Mastigocladus laminosus or for the $(\alpha \beta)_{3}$-trimers or the $(\alpha \beta)_{\sigma}$-hexamers of $A g m e n e l l u m ~ q u a d r u p l i c a t u m$, the new calculations predict excited state relaxation components with exponential time constants shorter than 1 ps. In fact, some of the interchromophore interactions are so strong that exciton coupling is probably the relevant mechanism of interaction. The langest exciton energy is calculated to be about $56 \mathrm{~cm}^{-1}$, for the interaction between the adjacent $a 84$ and $\beta 84$ chromophores of neighboring monomer units within the $(\alpha \beta)_{3}$-trimers or $(\alpha \beta)_{6}$-hexamers. An energy transfer model invoking a combination of pairwise exciton formation followed by slower Förster transfer steps is described.

\section{Introduction}

Excitation transfer in photosynthetic pigment antennas is responsible for the efficient collection of incident photons to provide the reaction centers with excitation energy. Typically, each reaction center is associated with an array of one hundred to several hundred antenna pigment molecules

Abbreviation: PC, C-phycocyanin.

Correspondence: K. Sauer, Laboratory of Chemical Biodynamics, Lawrence Berkeley Laboratory, University of California, Berkeley, CA 94720 , U.S.A. that transfer excitation to it with an efficiency of 95\% or greater and in a time that is much shorter than the lifetime (a few nanoseconds) of the excited state in the absence of trapping [1]. A model, known as the Pebble Mosaic Model, was developed to describe this excitation transfer process [2]. It is based on the knowledge that most photosynthetic pigments (chromophores) occur in welldefined associations with proteins and that these pigment proteins occur in organized arrays with respect to the embedded reaction centers in photosynthetically active membranes [3]. In the Pebble Mosaic Model excitation is considered to appear in collective excited states (excitons) that encompass all of the chromophores that interact with 
one another by exciton coupling within individual units of the pigment proteins. This exciton interaction is restricted, however, to chromophores that are coupled by energies greater than a few tens of $\mathrm{cm}^{-1}$. For longer-range transfer between pigment protein units, excitation is considered to migrate by a hopping process described by the inductive resonance mechanism of Förster [4]. This mechanism provides for effective collection of excitation energy by the reaction center from antenna molecules within a radius of about $10 \mathrm{~nm}$ for a single transfer step, and allows energy collection by multiple transfer steps over distances to several tens of nancmeters.

Evidence to support this description of the photosynthetic antenna has come largely from spectroscopic measurements. Exciton interactions are typically associated with absorption band splittings, conservative circular dichroism features and very rapid appearance of the excitation among all of the interacting chromophore molecules. Thermal relaxation among the associated exciton states appears to be faster than the picosecond limitation of most time-resolved absorption change or fluorescence measurements. Förster transfer, on the other hand, typically occurs at a rate that can be resolved by modern spectroscopic instrumentation. Evidence to support its presence includes the occurrence of an induction of emission from a 'fluorescer' molecule following the transfer of excitation from a 'sensitizer' molecule, in a picture developed for phycobiliproteins by Teaie and Dale [5]. This induction may be observed directly in cases where the sensitizer and fluorescer molecules exhibit significantly shifted spectral properties, or by time-resolved fluorescence depolarization when the wavelength dependences cannot be distinguished from one another. Analogous experiments have been carried out involving time-resolved absorption changes of either ground or excited state species $[6,7]$. For the situations where Förster transfer is dominant, the magnitudes of the exciton couplings that give rise to spectroscopic splittings are too small to detect. Intermediate cases may be difficult to characterize, however, because spectral band shifts and circular dichroism features induced by different protein environments among a set of chromophores can mimic the consequences of exciton band splitting.
MacColl et al. [8] have discussed the relevance of the exciton model to allophycocyanin. Furthermore, the transition region between the stronger coupling associated with exciton interaction and the weaker Förster inductive resonance has been investigated only theoretically by Kenkre and Knox [9], but has not been explored in a clear fashion experimentally.

Only recently have the structures of photosynthetic pigment protein complexes become known in sufficient detail to explore these questions of excitation transfer by direct calculations. Several studies have applied exciton theory to calculate the absorption and polarization spectra of a bacterial antenna complex [10] and of isolated reaction centers from Rhodopseudomonas viridis [11] whose structures are known through X-ray crystallographic determinations [12,13]. We have reported the results of initial efforts to calculate excitation transfer rates and excited state relaxation kinetics for $\mathbf{C}$-phycocyanin, a cyanobacterial antenna pigment protein that has been crystallized and solved by Schirmer et al. [14,15]. Based on the published preliminary structure parameters, we concluded that the Förster mechanism is sufficient to account for excitation transfer among the chromophores within C-phycocyanin [16].

Subsequent refinements of the crystallographic structure determination of $\mathrm{C}$-phycocyanin have led to a significantly improved definition of the chromophore locations and orientations, particularly for those of one of the chromophores ( $\beta 84)$ attached to the $\beta$-subunit peptide [17]. The principal parameters that govern the rate of excitation transfer (or the magnitude of exciton interaction) are (1) the distance of interchromophore separation, (2) the relative orientations of the respective transition dipole moments and (3) the strengths of the transition dipoles. The effective dielectric properties of the intervening space also play a role in modulating the interactions. The electric permittivity is usually assumed to be uniform between chromophores, but this is certainly not valid for groups that are very near one another; furthermore, the effective values may range over a factor of $\mathbf{1 . 5}$ in relative permittivity. The revised structures for C-phycocyanin show relatively small changes in the interchromophore separations (center-to-center distance); however, the orienta- 
tion of the $\beta 84$ chromophores is drastically altered. Because both the exciton and Förster theories involve dipole-dipole coupling, these alterations in chromophore orientation lead to pronounced changes in the calculated excitation transfer properties.

We have repeated the Forster transfer calculations using the revised coordinates from Schirmer et al. [17], and, in addition, have calculated pairwise exciton interactions among the chromophores. The results show large changes in several of the Förster rate constants and that exciton interactions can no longer be disregarded in analyzing the excitation transfer characteristics of C-phycocyanin, particularly for aggregates.

\section{Methods}

Rate constants for pairwise transfer between chromophores were calculated using the förster expression, as rescribed previously [16]. The overlap integrals of donor fluorescence and acceptor absorption were slightly revised from our previous calculation, based on an improved spectral resolution. The same radiative lifetime (1.5 ns) and hence different fluorescence yields consistent with the natural lifetimes were assumed for three chromophore types. New coordinates for the location and orientation of the chromophores of C-phycocyanin from Mastigocladus laminosus or from Agmenellum quadruplicatum were kindly provided in advance of publication by $\mathbf{T}$. Schirmer and $\mathbf{R}$. Huber. The crystals of C-phycocyanin from $A$. quadruplicatum contain $(\alpha \beta)_{6}$-hexamers, each of which consists of two $(\alpha \beta)_{3}$-trimers in a face-toface arrangement. The chromophoric arrangement in each of the two-component trimers is found to be very similar to that in C-phycocyanin from $M$. laminosus for most chromophore atoms. By contrast with our previous calculations, we have here assumed a refractive index of 1.34. (The authors would like to thank Prof. S.G. Boxer of Stanford University for calling attention to his analysis of the role and appropriate values to use for the refractive index in the Forster equations. Readers are referred to the informative Appendix of Moog et al. [18]). Because of the dependence of the Förster rate constants on the inverse fourth power of the refractive index, the result of this change is to increase all values of the rate constants by a factor of $(1.34 / 1.54)^{-4}=1.74$ relative to those calculated previously.

Excited-state relaxation kinetics can be calculated for the different dissociation states of physocyanin, assuming that the molecules retain their crystallographic geometry. We carried ou: such calculations using the kinetic model described previously and applied to the $\beta$-subunit, the $\alpha \beta$-monomer and the $(\alpha \beta)_{3}$-trimer for $C$ phycocyanin from both organisms and to the $(\alpha \beta)_{6}$-hexamer for the PC from $A$. quadruplicatum. For the trimers and hexamers the initial steps in the Euler method of calculating the relaxation were set to one-tenth the time interval used previously, starting with intervals of $1 \mathrm{fs}$, because of the much faster initiai relaxation attributable to the new inromophore coordinates for these aggregates. (Nevertheless, even with the larger steps, the calculations gave exponential decay times that diifer by less than $0.1 \%$ from the more refined ones.) Exponential decay constants were extracted from the calculated time dependence of the excited-state populations using a least-squares method of exponential analysis.

Estimates of the magnitude of exciton couplings among neighboring chromophores used a point dipole approximation for the transition moments. Transition dipole strengths were calculated for the $\alpha 84, \beta 84$ and $\beta 155$ chromophores by integrating over the visible (470-720 nm) absorption band in the deconvoluted spectra. Transition moment directions were estimated in the same manner as described by Schirmer et al. [14,15]; i.e., by assuming equal contributions from each of the chromophore atoms (carbon, nitrogen or oxygen) involved in the conjugated $\boldsymbol{m}$-electron system. All pairwise interactions were calculated using the simple point-dipole expression [19]

$V_{ \pm}= \pm D_{k} / \epsilon R^{3}$

where $D$ is the transition dipole strength (in debye $^{2}$ ), $\kappa$ is the orientation factor, $\epsilon$ is the relative permittivity (dielectric constant) and $R$ is the center-to-center separation of the chromophores. $D / \epsilon=\left(9.185 \cdot 10^{-39} / n\right) \int \varepsilon(v) / v \mathrm{~d} v$, in debye ${ }^{2}$, where the refractive index $n=1.34$, and absorptivities, $\varepsilon$, as a function of frequency, $v$, are de- 
termined from the spectroscopic deconvolution described in Sauer et al. [16]. $V_{ \pm}$, the exciton interaction energy, is one-half the expected spectroscopic splitting between the two exciton energy levels that result from the pairwise interactions.

\section{Results}

\section{Förster transfer calculations}

Calculated values for the rate constants (in $n^{-1}$ ) for excitation transfer between chromophores using the Förster theory are presented in Table I for PC from $A$. quadruplicatum and in Table II for PC from $M$. laminosus. In the case of $A$. quadruplicatum the values calculated from the superseded (old) geometry are included for comparison. (As mentioned above, these values differ from the ones in [16] by a factor of 1.74 owing to a corrected value for the refractive index.) The entries are orierced in riation to the aggregation state of the phycocyanins: the values for transfer between the two $\beta$-subunit chromophores ( $\beta 84$ and $\beta 155)$ are listed in the first line of data; for the $\alpha \beta$-monomer, these values plus those in the next two lines are appropriate; for larger aggregates the new entries under each heading need to be included together with those lying above them in the table. Within each cluster, the values are ordered roughly in order of decreasing importance. Among the severai symmetry-related equivalent transfers in aggregates, only the ones connecting to monomer 1 are listed. The chromophores involved in each pairwise interaction are designated in the columns headed $E$ or $F$, following the notation of Schirmer et al. [15]. Rate constants for 'forward transfer', from $E$ to $F$, are listed in the colurnns to the right; rate constants for 'back transfer', from $F$ to $E$, are listed in the columns to the left of the center of each line. The rates for forward transfer from $\alpha 84$ to $\beta 84$ are greater than those for back transfer between the same two chromophores; the differences between the forward- and back-transfer rates are even more pronounced for transfer from $\beta 155$ to either $\alpha 84$ or $\beta 84$, because of the less favorable spectral overlap. For the higher aggregates (trimers or hexamers) transfer beiween two chromophores of the same class are, of course, symmetric with respect to forward and back transfer.
Comparison of the results in Table I calculated for A. quadruplicatum for the old or the new coordinates shows some dramatic differences, particularly for the higher aggregates. For example, in the $(\alpha \beta)_{3}$-trimer or the $(\alpha \boldsymbol{\beta})_{6}$-hexamer, transfer from the $\alpha 84$ chromophore of one monomer to the adjacent $\beta 84$ chromophore of the next monomer in the same trimer is increased over 20 -fold in comparison with the previous calculation and is now by far the dominant transfer step in both of these aggregated species. As wo shall see, this has a pronounced effect on the calculated excited state relaxation kinetics. There is an even greater increase (nearly $\mathbf{4 0 0}$-fold) in the transfer rates between the $\alpha 84$ and $\beta 84$ chromophores within the same monomer; however, this has a less profound effect on the relaxation kinetics, because it is not the dominant relaxation step in any of the complexes. Additional consequences of the new results will be considered in the Discussion. Because of the close similarities in the structures determined for PC from $A$. quadruplicatum and $\boldsymbol{M}$. laminosus, the generalizations concerning the comparison of calculations between the former and the revised coordinates apply to the PC from the latter organism as well. The largest differences between the PC molecules from the two organisms concern transfer steps involving chromophore $\beta 155$. There are small but distinct alterations in its geometry, probably as a consequence of packing effects (Huber, R., personal communication).

Using the new values for the Förster transfer rate constants, we have calculated excited-state relaxation profiles for the various complexes. As discussed in our previous analysis, owing to the 3-fold symmetry of the protein, one expects the decays to be sums of three exponential components for each romnlex except for the separated $\alpha$ or $\beta$ subunits where only 1 or 2 exponentials, respectivelv, are expected. In each case one exponential decay component (the slowest) is determined solely by the intrinsic excited-state lifetime, which was input as $\mathbf{1 . 5}$ ns for our calculations. Values extractea from the calculated results for the lifetimes are presented in Table III. Although all of the decay components are modified to some extent in comparison with the calculated values published previously, the most dramatic change is in the rapid initial relaxation component 
TABLE I

RATE CONSTANTS FOR FÖRSTER EXCITATION TRANSFER IN C-PHYCOCYANIN FROM A. QUADRUPLICATUM

Rate constants calculated using the Förster inductive resonance transfer expression and spectroscopic overlap integrals, as described previously [16], but using a refractive index value $n=1.34$ [18! Chromonhore coordinates and orientation factors for PC are compared for the 'old' [15] and revised 'new' [17] values. Rate constants for forward $(E \rightarrow F)$ transfer are shown in tie columns at the right and for reverse $(F \rightarrow E)$ transfer in the columns at the left. For increasing extent of aggregation, values from the top of the table are to be included in a cumulative manner. For the $(\alpha \beta)_{3}$-trimer and $(\alpha \beta)_{6}$-hexamer, only the transfer steps with one terminus in monomer 1 ase listed; the others are related by symmetry. The nomenclature of Schirmer et al. [15] has been adopted: 1a84 $=$ chromophore $\alpha 84$ on monomer 1 .

\begin{tabular}{|c|c|c|c|c|}
\hline \multirow{2}{*}{\multicolumn{2}{|c|}{$\begin{array}{l}k\left(\mathrm{~ns}^{-1}\right) \\
\mathrm{F} \rightarrow \mathrm{E}\end{array}$}} & Chromophores & \multicolumn{2}{|l|}{$k\left(\mathrm{~ns}^{-1}\right)$} \\
\hline & & \multirow[t]{3}{*}{$\overline{\mathrm{E}}$} & \multirow{2}{*}{\multicolumn{2}{|c|}{$\begin{array}{l}\mathbf{E} \rightarrow \mathbf{F} \\
\text { Coordinates }\end{array}$}} \\
\hline Coordinates & & & & \\
\hline$\overline{\text { Old }}$ & New & & New & Old \\
\hline $\begin{array}{c}\beta \text {-Subunit } \\
6.3\end{array}$ & 7.6 & $1 \beta 155 \leftrightarrow 1 \beta 84$ & 34.4 & 28.5 \\
\hline $\begin{array}{c}\alpha \beta \text {-Monome } \\
0.028 \\
0.84\end{array}$ & $\begin{array}{c}10.6 \\
0.52\end{array}$ & $\begin{aligned} 1 \alpha 84 & \leftrightarrow 1 \beta 84 \\
1 \beta 155 & \leftrightarrow 1 \alpha 84\end{aligned}$ & $\begin{array}{c}13.9 \\
2.15\end{array}$ & $\begin{array}{l}0.037 \\
3.44\end{array}$ \\
\hline $\begin{array}{c}(\alpha \beta)_{3} \text {-Trim } \\
44.8\end{array}$ & 1160 & $\begin{array}{l}3 \alpha 84 \leftrightarrow 1 \beta 84 \\
1 \alpha 84 \leftrightarrow 2 \beta 84\end{array}$ & 1527 & 59.0 \\
\hline 46.2 & 8.8 & $\begin{array}{l}2 \beta 84 \leftrightarrow 1 \beta 84 \\
3 \beta 84 \leftrightarrow 1 \beta 84\end{array}$ & 8.8 & 46.2 \\
\hline 0.138 & 1.62 & $\begin{array}{l}2 \alpha 84 \leftrightarrow 1 \beta 84 \\
1 \alpha 84 \leftrightarrow 3 \beta 84\end{array}$ & 2.14 & 0.18 \\
\hline 4.1 & 0.58 & $\begin{array}{l}2 \beta 155 \leftrightarrow 1 \alpha 84 \\
1 \beta 155 \leftrightarrow 3 \alpha 84\end{array}$ & 2.4 & 16.9 \\
\hline $0.4 \overline{2}$ & 1.25 & $\begin{array}{l}2 a 84 \leftrightarrow 1 \alpha 84 \\
3 a 84 \leftrightarrow 1 \alpha 84\end{array}$ & 1.25 & 0.42 \\
\hline 1.37 & 0.18 & $\begin{array}{l}3 \beta 155 \leftrightarrow 1 \beta 84 \\
1 \beta 155 \leftrightarrow 2 \beta 84\end{array}$ & 0.82 & 6.2 \\
\hline 0.35 & 0.131 & $\begin{array}{l}2 \beta 155 \leftrightarrow 1 \beta 84 \\
1 \beta 155 \leftrightarrow 3 \beta 84\end{array}$ & 0.59 & 1.55 \\
\hline 0.37 & 0.29 & $\begin{array}{l}2 \beta 155 \leftrightarrow 1 \beta 155 \\
3 \beta 155 \leftrightarrow 1 \beta 155\end{array}$ & 0.29 & 0.37 \\
\hline 0.0131 & 0.023 & $\begin{array}{l}3 \beta 155 \leftrightarrow 1 \alpha 84 \\
1 \beta 155 \leftrightarrow 2 \alpha 84\end{array}$ & 0.092 & $0.0 \leqslant 4$ \\
\hline$(\alpha \beta)_{6}-\mathrm{Hexa}$ & mer & & & \\
\hline 185.4 & 391.0 & $4 a 84 \leftrightarrow 1 \alpha 84$ & 391.0 & 185.4 \\
\hline $\begin{array}{r}174.8 \\
5.0\end{array}$ & $\begin{array}{r}186.3 \\
16.7\end{array}$ & $\begin{array}{l}6 \beta 155 \leftrightarrow 1 \beta 155 \\
6 \beta 155 \leftrightarrow 1 \alpha 84 \\
1 \beta 155 \leftrightarrow 6 \alpha 84\end{array}$ & $\begin{array}{r}186.3 \\
68.8\end{array}$ & $\begin{array}{r}174.8 \\
20.7\end{array}$ \\
\hline 1.24 & 30.9 & $\begin{array}{l}6 \alpha 84 \leftrightarrow 1 \beta 84 \\
1 \alpha 84 \leftrightarrow 6 \beta 84\end{array}$ & 40.7 & 1.64 \\
\hline
\end{tabular}

TABLE 1 (continued)

\begin{tabular}{|c|c|c|c|c|}
\hline \multirow{2}{*}{\multicolumn{2}{|c|}{$\begin{array}{l}k\left(\mathrm{~ns}^{-1}\right) \\
\mathrm{F} \rightarrow \mathrm{E}\end{array}$}} & Chromophores & \multirow{2}{*}{\multicolumn{2}{|c|}{$\frac{k\left(\mathrm{~ns}^{-1}\right)}{\mathrm{E} \rightarrow \mathrm{F}}$}} \\
\hline & & \multirow[t]{3}{*}{$\overline{\mathbf{E}}$} & & \\
\hline Coordinate & & & \multicolumn{2}{|c|}{ Coordinates } \\
\hline Old & New & & New & Old \\
\hline 10.9 & 10.6 & $6 \beta 84 \leftrightarrow 1 \beta 84$ & 10.6 & 10.9 \\
\hline 0.45 & 2.04 & $\begin{array}{l}6 \beta 155 \leftrightarrow 1 \beta 84 \\
\mathrm{i} \beta 155 \leftrightarrow 6 \beta 84\end{array}$ & 9.2 & 2.01 \\
\hline 53.6 & 9.0 & $5 \beta 84 \leftrightarrow 1 \beta 84$ & 9.0 & 53.6 \\
\hline 10.2 & 4.5 & $4 \beta 84 \leftrightarrow 1 \beta 84$ & 4.5 & 10.2 \\
\hline 0.244 & 0.83 & $\begin{array}{l}5 \beta 155 \leftrightarrow 1 \beta 84 \\
1 \beta 155 \leftrightarrow 5 \beta 84\end{array}$ & 3.8 & 1.11 \\
\hline 0.045 & 0.59 & $\begin{array}{l}4 \alpha 84 \leftrightarrow 1 \beta 84 \\
1 \alpha 84 \leftrightarrow 4 \beta 84\end{array}$ & 1.31 & 0.059 \\
\hline 0.58 & 0.94 & $6 \alpha 84 \leftrightarrow 1 \alpha 84$ & 0.94 & 0.58 \\
\hline 0.29 & 0.176 & $\begin{array}{l}4 \beta 155 \leftrightarrow 1 \beta 84 \\
1 \beta 155 \leftrightarrow 4 \beta 84\end{array}$ & 0.79 & 1.31 \\
\hline 0.033 & 0.54 & $\begin{array}{l}5 a 84 \leftrightarrow 1 \beta 84 \\
1 \alpha 84 \leftrightarrow 5 \beta 84\end{array}$ & 0.72 & 0.044 \\
\hline 0.85 & 0.134 & $\begin{array}{l}4 \beta 155 \leftrightarrow 1 \alpha 84 \\
1 \beta 155 \leftrightarrow 4 \alpha 84\end{array}$ & 0.55 & 3.5 \\
\hline 0.0009 & 0.127 & $\begin{array}{l}5 \beta 155 \leftrightarrow 1 \alpha 84 \\
1 \beta 155 \leftrightarrow 5 \alpha 84\end{array}$ & 0.52 & 0.0033 \\
\hline 0.134 & 0.37 & $5 \alpha 84 \leftrightarrow 1 \alpha 84$ & 0.37 & 0.134 \\
\hline 0035 & 0.0112 & $S \beta 1 S S \leftrightarrow 1 \beta 15 S$ & 0.0112 & 0.035 \\
\hline 0.045 & 0.0094 & $4 \beta 155 \leftrightarrow 1 \beta 155$ & 0.0094 & 0.045 \\
\hline
\end{tabular}

seen in the calculated results for the $(\alpha \beta)_{3}$-trimer or $(\alpha \beta)_{6}$-hexamer. These expected lifetimes are now less than 1 ps, whereas they were greater than 10 ps previously. A word of caution needs to be stated concerning putting too much emphasis on these subpicosecond calculated exponential component lifetimes, however. As we will show presently, it is likely that the Förster mechanism of excitation transfer breaks down for these closely coupled chromophores and that exciton coupling has to be considered to play a role in excited state delocalization. If anything, however, this is expected to speed up the initial relaxation from that predicted by Förster theory, which will give oniy lower limits for the rate constants.

\section{Exciton calculations}

The results of the pairwise exciton calculations are presented in Table IV. These have been carried out assuming that the energies of the transitions are identical for the three chromophore classes, which is reasonable for the $\alpha 84$ and $\beta 84$ chromophores showing the strongest interactions. 


\section{TABLE II}

RATE CONSTANTS FOR FÖRSTER EXCITATION TRANSFER IN C-PHYCOCYANIN FROM MASTIGOCLADUS LAMINOSUS

Rate constants calculated using the Förster inductive resonance transfer expression and spectroscopic overlap integrals, as described previously [16], but using a refractive index value $n=\mathbf{i} .3+[18]$. Chromophore coordinates and orientation factors for PC are taken from Schirmer et al. [17]. Organization as in Table I.

\begin{tabular}{|c|c|c|}
\hline \multirow{2}{*}{$\begin{array}{l}k\left(\mathrm{~ns}^{-1}\right) \\
\mathrm{F} \rightarrow \mathrm{E}\end{array}$} & Chromophores & \multirow{2}{*}{$\frac{k\left(\mathrm{~ns}^{-1}\right)}{\mathrm{E} \rightarrow \mathrm{F}}$} \\
\hline & $E$ & \\
\hline $\begin{array}{c}\beta \text {-Subunit } \\
9.1\end{array}$ & $1 \beta 155 \leftrightarrow 1 \beta 84$ & 41.0 \\
\hline $\begin{array}{c}\alpha \beta \text {-Mono } \\
11.1 \\
0.99\end{array}$ & $\begin{array}{l}1 \alpha 84 \leftrightarrow 1 \beta 84 \\
1 \beta 155 \leftrightarrow 1 \alpha 84\end{array}$ & $\begin{array}{c}14.6 \\
4.06\end{array}$ \\
\hline $\begin{array}{l}(\alpha \beta)_{3} \text {-Trir } \\
1312.0\end{array}$ & $3 \alpha 84 \leftrightarrow 1 \beta 84$ & 1727.0 \\
\hline 10.4 & $\begin{array}{l}1 \alpha 84 \leftrightarrow 2 \beta 84 \\
2 \beta 84 \leftrightarrow 1 \beta 84 \\
3 \beta 84 \leftrightarrow 1 \beta 84\end{array}$ & 10.4 \\
\hline 1.77 & $\begin{array}{l}2 \alpha 84 \leftrightarrow 1 \beta 84 \\
1 \alpha 84 \leftrightarrow 3 \beta 84\end{array}$ & 2.33 \\
\hline 1.39 & $\begin{array}{l}2 \beta 155 \leftrightarrow 1 \alpha 84 \\
1 \beta 155 \leftrightarrow 3 \alpha 84\end{array}$ & 5.7 \\
\hline 1.29 & $\begin{aligned} 2 \alpha 84 & \leftrightarrow 1 \alpha 84 \\
3 \alpha 84 & \leftrightarrow 1 \alpha 84\end{aligned}$ & 1.29 \\
\hline 0.159 & $\begin{array}{l}3 \beta 155 \leftrightarrow 1 \beta 84 \\
1 \beta 155 \leftrightarrow 2 \beta 84\end{array}$ & 0.72 \\
\hline 0.204 & $\begin{array}{l}2 \beta 155 \leftrightarrow 1 \beta 84 \\
1 \beta 155 \leftrightarrow 3 \beta 84\end{array}$ & 0.92 \\
\hline 0.36 & $\begin{array}{l}2 \beta 155 \leftrightarrow 1 \beta 155 \\
3 \beta 155 \leftrightarrow 1 \beta 155\end{array}$ & 0.36 \\
\hline 0.045 & $\begin{array}{l}3 \beta 155 \leftrightarrow 1 \alpha 84 \\
1 \beta 155 \leftrightarrow 2 \alpha 84\end{array}$ & 0.183 \\
\hline
\end{tabular}

In the resolved PC spectra, the separation (160 $\mathrm{cm}^{-1}$ ) of the absorption band maxima of these chromophores is small compared with the absorption bandwidths (FWHM $850 \mathrm{~cm}^{-1}$ ). The maximum interaction energy of about $56 \mathrm{~cm}^{-1}$ $(1 \alpha 84 / 2 \beta 84)$ in Table IV cannot be ignored, and the relative contribution of excitonic or some intermediate form of coupling needs to be considered seriously in addition to Förster resonance transfer for at least the trimers and hexamers of PC.

Pursuing this pairwise exciton model, it is possible to calculate the transition moment directions for the two resulting exciton states and to estimate their relative dipole strengths. Because of the $C_{3}$ symmetry axis, the three chromophore pairs will result in transitions that exhibit axial symmetry; i.e., they will collectively be degenerate in the plane perpendicular to the $\boldsymbol{C}_{3}$ symmetry axis for $(\alpha \beta)_{3}$-trimers or $(\alpha \beta)_{6}$-hexamers. Calculations using the chromophore coordinates and transition moment directions of Schirmer et al. ([17], Table 9) indicate that the long wavelength exciton absorption oscillator should lie within $10^{\circ}$ of this plane, with only a small component along the $C_{3}$ symmetry axis. The short wavelength exciton state oscillator, which will be perpendicular to the long wavelength component, should be at about $45^{\circ}$ to the plane and, thus, is expected to have a significant contribution along the symmetry axis.

The exciton picture presented here is, of course, based on a crude model involving only pairwise interactions, so it offers only an indication that this problem needs to be addressed more rigorously. For aggregates containing numbers of chromophores, a proper analysis needs to calculate the

\section{TABLE III}

EXCITED STATE EXPONENTIAL DECAY TIME CONSTANTS CALCULATED FOR FÖRSTER EXCITATION TRANSFER IN C-PHYCOCYANIN

Calculated values listed are time constants in ps for the fast $\left(\tau_{1}\right)$ and intermediate $\left(\tau_{2}\right)$ components of a three-exponential analysis, with $k_{\mathrm{F}}$ input as $0.67 \mathrm{~ns}^{-1}$ for the rate constant of radiative and non-radiative decay corresponding to $\tau_{3}$.

\begin{tabular}{lcc}
\hline & $\tau(\mathrm{ps})$ & \\
\cline { 2 - 3 } & A. quadruplicatum & M. laminosus \\
\hline $\boldsymbol{\beta}$-Subunit & 24 & 20 \\
$\tau_{1}$ & 2400 & 1500 \\
$\tau_{3}$ & 1500 & \\
$\alpha \beta$-Monomer & & 18 \\
$\tau_{1}$ & 21 & 41 \\
$\tau_{2}$ & 45 & 1500 \\
$\tau_{3}$ & 1500 & \\
$(\alpha \beta)_{3}$-Trimer & & 0.33 \\
$\tau_{1}$ & 0.37 & 17 \\
$\tau_{2}$ & 22 & 1500 \\
$\tau_{3}$ & 1500 & \\
$(\alpha \beta)_{6}$-Hexamer & \\
$\tau_{1}$ & 0.36 & \\
$\tau_{2}$ & 7.5 & \\
$\tau_{3}$ & 1500 & \\
\hline
\end{tabular}


TABL.E IV

EXCITON ENERGIES OF PAIRWISE INTERACTION CALCULATED FOR CHROMOPHORES OF C-PHYCOCYANIN FROM A. QUADRUPLICATUM

Exciton energies calculated from the relation $V_{t}= \pm D k / \epsilon R^{3}$, with orientation factors, $\kappa$, and interchromophore distances, $R$, taken from Schirmer, et al. [17]. $D$, dipole strength and $\epsilon$, local dielectric constant.

\begin{tabular}{|c|c|c|c|c|c|c|c|}
\hline \multicolumn{2}{|l|}{$\beta$-Subunit } & \multicolumn{2}{|l|}{$\alpha \beta$-Monomer } & \multicolumn{2}{|l|}{$(\alpha \beta)_{3}$-Trimer } & \multicolumn{2}{|l|}{$(\alpha \beta)_{6}$-Hexamer } \\
\hline & $\begin{array}{l}V_{ \pm} \\
\left(\mathrm{cm}^{-1}\right)\end{array}$ & & $\begin{array}{l}V_{ \pm} \\
\left(\mathrm{cm}^{-1}\right)\end{array}$ & & $\begin{array}{l}V_{ \pm} \\
\left(\mathrm{cm}^{-1}\right)\end{array}$ & & $\begin{array}{l}V_{ \pm} \\
\left(\mathrm{cm}^{-1}\right)\end{array}$ \\
\hline \multirow[t]{31}{*}{$1 \beta 155 / 1 \beta 84$} & 7.3 & $1 \alpha 84 / 1 \beta 84$ & 5.3 & $\begin{array}{l}1 a 84 / 2 \beta 84 \\
1 \alpha 84 / 3 \beta 84\end{array}$ & 56 & $1 \alpha 84 / 4 a 84$ & 32.0 \\
\hline & & $1 \beta 155 / 1 \alpha 84$ & 1.9 & & & $1 \beta 155 / 6 \beta 155$ & 23.8 \\
\hline & & & & $1 \beta 84 / 2 \beta 84$ & 4.3 & & \\
\hline & & & & $1 \beta 84 / 3 \beta 84$ & & $\begin{array}{l}1 \alpha 84 / 6 \beta 155 \\
1 \beta 155 / 6 \alpha 84\end{array}$ & 10.8 \\
\hline & & & & $1 \beta 84 / 2 \alpha 84$ & 2.1 & & \\
\hline & & & & $1 \alpha 84 / 3 \beta 84$ & & $1 \alpha 84 / 6 \beta 84$ & 9.1 \\
\hline & & & & & & $1 \beta 84 / 6 \alpha 84$ & \\
\hline & & & & $1 \alpha 84 / 2 \beta 155$ & 2.0 & & \\
\hline & & & & $1 \beta 155 / 3 \alpha 84$ & & $1 \beta 84 / 6 \beta 84$ & 4.7 \\
\hline & & & & $\begin{array}{l}1 \alpha 84 / 2 \alpha 84 \\
1 \alpha 84 / 3 \alpha 84\end{array}$ & 1.8 & $1 \beta 84 / 5 \beta 84$ & 4.3 \\
\hline & & & & & & $1,884,6 \beta 155$ & 3.8 \\
\hline & & & & $1 \beta 155 / 2 \beta 84$ & 1.1 & $1 \beta 155 / 6 \beta 84$ & \\
\hline & & & & $1 \beta 84 / 3 \beta 155$ & & & \\
\hline & & & & & & $1 \beta 84 / 4 \beta 84$ & 3.1 \\
\hline & & & & $1 \beta 84 / 2 \beta 155$ & 1.0 & & \\
\hline & & & & $1 \beta 155 / 3 \beta 84$ & & $1 \beta 84 / 5 \beta 15 S$ & 2.4 \\
\hline & & & & & & $1 \beta 155 / 5 \beta 84$ & \\
\hline & & & & $1 \beta 155 / 2 \beta 155$ & 1.0 & & \\
\hline & & & & $1 \beta 155 / 3 \beta 155$ & & $\begin{array}{l}1 \alpha 84 / 4 \beta 84 \\
1 \beta 84 / 4 \alpha 84\end{array}$ & 1.6 \\
\hline & & & & $1 \alpha 84 / 3 \beta 155$ & 0.4 & & \\
\hline & & & & $1 \beta 155 / 2 \alpha 84$ & & $1 \alpha 84 / 6 a 84$ & 1.6 \\
\hline & & & & & & $1 \alpha 84 / 5 \beta 84$ & 1.2 \\
\hline & & & & & & $1 \beta 84 / 5 a 84$ & \\
\hline & & & & & & $1 \beta 84 / 4 \beta 155$ & 1.1 \\
\hline & & & & & & $1 \beta 15 S / 4 \beta 84$ & \\
\hline & & & & & & $1 \alpha 84 / 5 a 84$ & 1.0 \\
\hline & & & & & & $1 \alpha 84 / 5 \beta 155$ & 1.0 \\
\hline & & & & & & $1 \beta 155 / 4 \alpha 84$ & \\
\hline & & & & & & $\begin{array}{l}1 a 84 / 5 \beta 155 \\
1 \beta 155 / 4 \alpha 84\end{array}$ & 1.0 \\
\hline & & & & & & $1 \beta 155 / 5 \beta 155$ & 0.2 \\
\hline & & & & & & $1 \beta 155 / 6 \beta 155$ & 0.15 \\
\hline
\end{tabular}

states resulting from interactions among the full set of chromophores. Our approximation may, fortuitously, be not so bad for the particular case of PC, because the interaction between particular pairs of chromophores, adjacent $\alpha 84 / \beta 84$, is significantly larger than any other. We tested this 


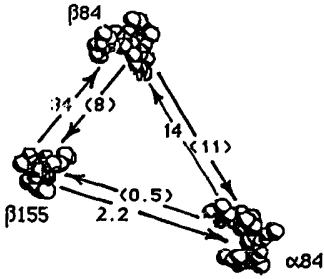

$\alpha \beta$ - Monomer
$8 \% 3 \beta 155$

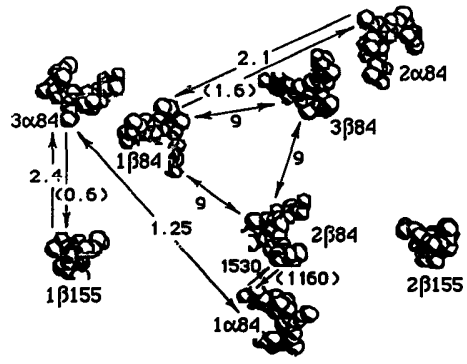

$(\alpha \beta)_{3}-$ Trimer

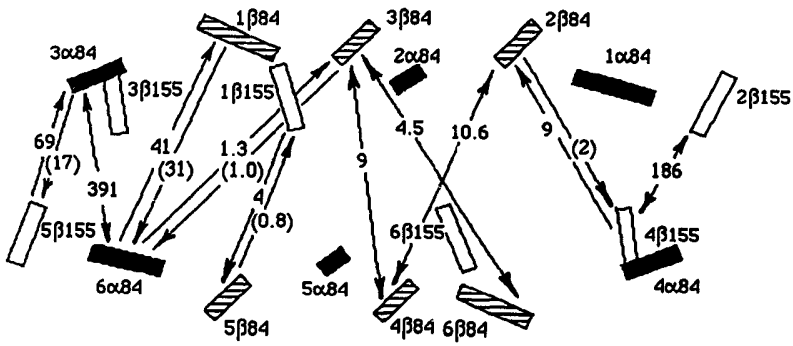

$(\alpha \beta)_{6}$ - Hexamer

Fig. 1. Diagrams of the arrangement of chromophores and values of Förster resonance transfer rate constants in $\mathrm{ns}^{-1}$ for the most important steps $\left(k>1 \mathrm{~ns}^{-1}\right)$ in C-phycocyanin from $A$. quadruplicatum, based on the revised structure of Schirmer et al. [17]. We thank these authors for providing the coordinates in advance of publication. The diagrams for $\alpha \beta$-monomer, $(\alpha \beta)_{3}$-trimer and $(\alpha \beta)_{\kappa}$-hexamer should be considered in a cumulative fashion; i.e., only the additional transfer steps not present in the simpler complex(es) are shown for the aggregated forms. For transfers between dissimilar chromophores, the slower rate for uphill transfer is given in parentheses after the faster raie for downhill transfer.

approximation by calculating some three-chromophore interactions using a formulation derived by Ebrey et al. [20] making use of the $C_{3}$ symmetry of like chromophores in the $(\alpha \beta)_{3}$-trimers. For the three-chromophore coupling of the $\alpha 84$ chromophores, the energy of interaction was calculated to 
be less than $1 \mathrm{~cm}^{-1}$. Similar small values (under 1 $\mathrm{cm}^{-1}$ ) were obtained for the sets of three $\beta 84 \mathrm{or}$ three $\beta 155$ chromophores. The small interactions among the $\alpha 84$ or $\beta 155$ chromophores result from the relatively large distances of separation of the chromophores from one another, 6.9 and $7.0 \mathrm{~nm}$, respectively. For the $\beta 84$ chromophores the smaller distance $(3.5 \mathrm{~nm})$ is compensated by a smaller transition dipole moment and by a relatively unfavorable orientation factor.

\section{Discussion}

In our initial efforts to calculate the path and kinetics of excitation transfer and fluorescence depolarization in PC we invoked the Förster inductive resonance transfer mechanism and applied it to the recently published crystallographic coordinates for PC from $M$. laminosus [14] and, in particular, from A. quadruplicatum [15]. Based on the results of these calculations we concluded that the Förster mechanism was sufficient to account for the excitation transfer rates expected and, furthermore, that there was rather good agreement between the calculated excited state decay kinetics and the available experimental data on fluorescence lifetimes and depolarization kinetics for these pigment proteins.

Refinement of the X-ray crystallographic structure has resulted in changes in the reported positions and especially in the orientations of several of the chromophores. The greatest consequences result for the $\beta 84$ chromophores, which are closest to the space at the core of the ring-like structure of the trimers. In the previous structure analyses, electron density was poorly defined for the pyrrole rings $\mathbf{C}$ and $\mathbf{D}$ that protrude into this inside space (the rings that are farthest from the location of covalent attachment to the protein at ring $A$ ). In the new structures electron density is well defined for each of the three types of chromophore, so that little further change is expected in subsequent refinements.

Few of the new interchromophore distances differ by as much as $12 \%$ from the previous values; however, because of the inverse sixth-power dependence on distance, changes of even this small magnitude can alter the calculated rate constants using the Forster theory by a factor of nearly 2 . Nevertheless, the largest consequences occur be- cause of revised values of the chromophore orientations, which result in altered $\kappa^{2}$ (orientation factor) vaiues, in one case by a factor of 175 . Not every case where such large changes occur in $\kappa^{2}$ results in detectable changes in the excited-state relaxation kinetics, howevsr. Significantly, for the chroinophore pair in trimers already showing the strongest interactions based on the previous structures, both the radius decrease and the $\kappa^{2}$ increase combine to make it extraordinariiy rapid for the new structures.

The pattern of excitation transfer within PC at the different levels of aggregation may be followed by reference to Fig. 1, where a model of the chromophore organization is developed in terms of the most important excitation-transfer steps.

\section{$\beta-S u b u n i t$}

With only two chromophores per $\boldsymbol{\beta}$-subunit polypeptide, this is the simplest case where excitation transfer can be investigated in PC. Inspection of the rate constants in Table I shows that there has been an increase of about $20 \%$ in the calculated transfer rates between the $\beta 155$ and the $\beta 84$ chromophores. This results in an expected exponential decay component of about 20 ps for the $\beta$-subunits of PC from either $\boldsymbol{M}$. laminosus or $\boldsymbol{A}$. quadruplicatum. While this is appreciably shorter than the experimental value of $190 \mathrm{ps}$ reported by Hefferle et al. [21], it should be noted that $\beta$-subunits exist as dimers in the isolated state (unpublished results). In general, such increased aggregation would be expected to shorten rather than lengthen the excited state relaxation times, unless there is a major conformational change that affects the excitation transfer rates within each subunit.

\section{$\boldsymbol{\alpha} \boldsymbol{\beta}$-Monomer}

Excitation transfer between the $\alpha 84$ and $\beta 84$ chromophores is found (Table I) to increase nearly 400-fold for the new coordinates in comparison with the previous ones. What was previously a negligible excitation transfer path now constitutes the dominant path of excitation exchange involving the $\alpha-84$ chromophores. The previous calculations indicated that energy flow from the $\alpha 84$ to the $\beta 84$ chromophore would go principally by the over-the-hill route through the $\beta 155$ chromophore. That is no longer the case for the new chromo- 
phore coordinates, where the direct exchange between $\alpha 84$ and $\beta 84$ is the more probable route. Nevertheless, excitation transfer from $\beta 155$ to $\beta 84$ chromophores remains the fastest of all in the $\alpha \beta$-monomer. This is the dominant factor in determining the most rapid exponential relaxation component of about 20 ps (Table III), which is not much different from that calculated for the isolated $\beta$-subunit but somewhat shorter than the experimental value of 47 ps reported for the $\alpha \beta$ monomer by Holzwarth et al. [22]. A comparable fast decay of about 57 ps based on ground-state bleaching recovery studies at $590 \mathrm{~nm}$ on PC monomers from $\boldsymbol{M}$. laminosus has been reported by Gillbro et al. [23]. The intermediate decay, expected to be about 45 ps (Table III), is much faster than that calculated previously $(700 \mathrm{ps})$, owing principally to the much faster direct transfer between the $\alpha 84$ and $\beta 84$ chromophores. Because these two calculated lifetimes are rather close to one another, this may present problems in resolving them experimentally. It could account for the experimental observation of only a single, but wavelengtli-dependent, exponential fluorescence decay component in this time region [23,24] and with a value intermediate between the calculated ones. The transient absorption studies [23] also show an increase in lifetime of the fastest decay to $178 \mathrm{ps}$ at longer wavelength (635-640 $\mathrm{nm}$ ), which is, however, much slower than the values calculated here or observed by fluorescence relaxation. Better wavelength-resolved kinetic measurements of excited-state relaxations of the $\boldsymbol{\alpha} \boldsymbol{\beta}$-monomer preparations would certainly be of value in addressing these issues.

Inspection of the results in Table IV shows that none of the chromophores is expected to be coupled by an exciton energy of greater than about 5 $\mathrm{cm}^{-1}$ for the $\alpha \beta$-monomer or the $\beta$-subunit. Because this is small compared with the bandwidth of the major electronic transitions in phycobiliproteins, little contribution is expected from exciton interaction within these unaggregated species and no spectroscopic splittings should be detectable experimentally.

\section{$(\boldsymbol{\alpha} \beta)_{3}$-Trimer}

Analysis of the crystal structure of PC shows that the $(\alpha \beta)_{3}$-trimer units consist of a ring of $\alpha \beta$-monomers in head-to-tail contact, with a $\mathrm{C}_{3}$ rotation axis passing through the center and perpendicular to the ring. This arrangement brings the $\alpha 84$ chromophore of one monomer unit into close proximity $(2.1 \mathrm{~nm})$ with the $\beta 84$ chromophore of the neighboring monomer. Despite the fact that these are the chromophores that are nearest to one another in the $(\alpha \beta)_{3}$-trimer, the previous analyses indicated the likelihood of an unfavorable orientation, which resulted in a transfer rate only about twice as large as that between the $\beta 155$ and $\beta 84$ chromophores within each monomer unit. The now coordinates show that this orientation factor is, in fact, quite favorable. Together with a somewhat shorter center-to-center distance, this results in an increase of more than 25-fold in the calculated excitation transfer rates between the $\alpha 84$ and $\beta 84$ chromophores of adjacent monomer units within the $(\alpha \beta)_{3}$-trimer. While this was marginally the fastest transfer step resulting from the previous calculations, the new results indicate that it should be overwhelmingly dominant. What was previously the second-fastest transfer process, direct transfer among the three $\beta 84$ chromophores of the $(\alpha \hat{\beta})_{3}$-trimer, is actuaily decreased significantly in the new results. The dominance of transfer between the adjacent $\alpha 84$ and $\beta 84$ chromophores remains true also in the $(\alpha \beta)_{6}$-hexamer, as can be seen from the rate constants presented in Table $I$.

Analysis of the excited state relaxation kinetics as a sum of exponentials based on the Forrster transfer mechanism indicates that the fastest component for the $(\alpha \beta)_{3}$-trimer should have a time constant less than 1 ps. This is much faster than the reported experimental values of 20 to 36 ps from fluorescence relaxation $[22,25]$ or the values of 21 to 48 ps from absorption transient measurements $[23,26,27]$. In fact, the calculated fast relaxation times are at [26] or beyond [24] the expected limits of time resolution for the experimental results reported to date $[24,26]$.

The short interchromophore distances and favorable orientation factors which result in short transfer times calculated on the basis of the Förster mechanism will also produce large values for the exciton interaction energies. As is seen in Table IV, a value of $56 \mathrm{~cm}^{-1}$ is calculated for the strongest $\alpha 84$ to $\beta 84$ interaction in the $(\alpha \beta)_{3}$-tri- 

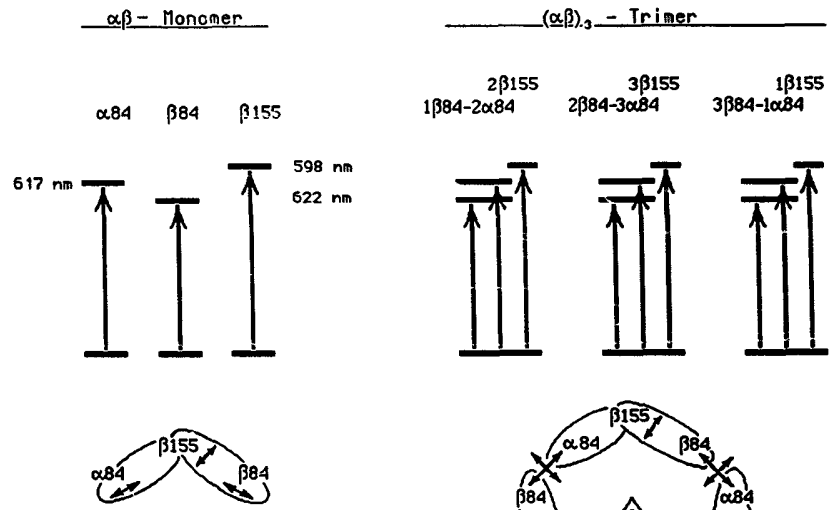

$598 \mathrm{np}$

$616 \mathrm{~nm}$

623 na

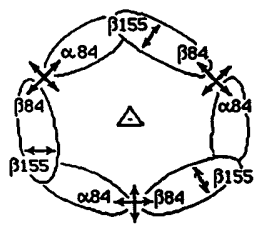

Fig. 2. Proposed model for exciton interaction in the $(\alpha \beta)$-trimer of PC. In the $\alpha \beta$ monomer (leit side) the $\alpha 84, \beta 84$ and $\beta 155$ chromophores are sufficiently separated that no significant exciton interaction is present. The three energy levels deduced on the basis of spectral deconvolution can each be associated with an individual chromophore. In the $(\alpha \beta)_{3}$-trimer (right side) stronger interaction ( $50-100 \mathrm{~cm}^{-1}$ ) between the $\alpha 84$ and $\beta 84$ chromophores of adjacent 'monomer' components results in a largely pairwise delocalization of the excitation between the two chromophores. Two exciton states result from this interaction. (The wavelength values given for the trimer result from an improved deconvolution of the spectra. They are slightly different from those of the monomer, which were previcusly assumed to apply also to the trimor [15].) Because the chromophore uransitivin moment directions

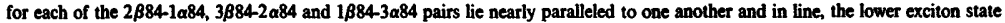
should have the greater absorption intensity and be polarized along the line of centers of the chromophores. The upper exciton state should show weaker absorption and be oriented perpendicular to the first. The low-energy transition moment direction should be more perpendicular than parallel to the $\mathrm{C}_{3}$ symmetry axis, but the higher energy transition should have a relatively large component along the $\mathbf{C}_{3}$ axis. This model is based on ideas developed by MacColl et al. [8] to interpret the spectra of allophycocyanin.

mer. The spectroscopic splitting $\left(2 V_{ \pm}=112 \mathrm{~cm}^{-1}\right)$ expected from this interaction is comparable with the separation $\left(160 \mathrm{~cm}^{-1}\right)$ between the 'resolved' absorption band components assigned to the $\alpha 84$ and $\beta 84$ chromophores. Thus, we may expect the exciton interaction to result in collective excited states involving these two chromophores. This is illustrated in an energy level diagram modeled for PC in Fig. 2, where the excited states are considered to be delocalized over the adjacent $\alpha 84$ and $\beta 84$ chromophores in the trimers (and hexamers). Assuming that the exciton coupling is limited to pairwise interaction, which appears to be a realis- tic model, this will result in two electronic excited states with energies $E \pm V_{ \pm}$for each $\alpha 84 / \beta 84$ pair. The lower of these two energy levels will result in emission or energy transfer to other chromophores. Relaxation from the upper to the lower state should be fast - probably in the picosecond time domain - but this remains to be explored experimentally. A possible interpretation of fluorescence kinetics in terms of this exciton model will be considered presently.

The rate-limiting steps in excited state relaxation for the $(\alpha \beta)_{3}$-trimer then become those involved in excitation transfer from the $\beta 155$ chro- 
mophore. Although the rate constants presented in Tables $I$ and II indicate that the transfer between the $\beta 155$ and the $\beta 84$ chromophore within the same $\beta$-subunit is still the dominant path of excitation loss from $\beta 155$ in the $(\alpha \beta)_{3}$-trimer, these numbers are no longer appropriate to the model involving exciton coupling between $\alpha 84$ and $\beta 84$ chromophores. Nevertheless, in the pure resonance-transfer (Förster) model, the exporiential decay component with the 20 ps lifetime reflects primarily this loss of excitation from the $\beta 155$ chromophore. It is interesting that in the recent report from Holzwarth et al. [22], where the decay components of PC fluorescence are wavelength resolved, the 35 ps decay component for the $(\alpha \beta)_{3}$-trimer emission does appear to result from excitation transfer from the shortest wavelength absorbing chromophore, presumably $\boldsymbol{\beta} 155$. A similar conclusion was reached based on the wavelength dependence of the absorption transient studies [23]. The wavelength dependence of the longer lifetime (120 and 600 ps) components cannot be interpreted meaningfully using either the pure Förster or the combined Förster/exciton modeīs presented here. The Förster model results in no additional decay shorter than the 1500 ps component that results from the intrinsic fluorescence decay of an isolated PC chromophore; presumably this latter is the fourth component reported from the analysis of the experimental fluorescence relaxation ( $1320 \mathrm{ps)}$ ) or the slowest of the absorption transients (1200 ps). For the combined Förster/exciton model this slow decay will occur predominantly from the lowest energy exciton states with kinetics that cannot be calculated adequately based on the information currently available.

An alternative model for excited state relaxation involving $\alpha 84 / \beta 84$ pairwise exciton interaction needs to be considered. Although the excitation is distributed among the exciton-coupled chromophores immediately upon absorption of a photon and the process is therefore too fast to resolve experimentally, the relaxation from the upper exciton state to the lower state may not be so rapid. If this relaxation were to occur in the region of $10-30 \mathrm{ps}$, then it would give the appearance of $s$ to $f$ transfer - in the sense that excitation into a shorter wavelength absorption band would result in stimulation of fluorescence from a lower energy state and with a short onset time or induction for the latter. In the exciton model, the excitation in each exciton state is not 'localized' on either of these chromophores, as is explicitly the case for the s-to-f transfer model, however. The rate of relaxation from the upper to the lower exciton states is not readily amenable to calculation. Although the two states are orthogonal to one another, they would be expected to mix by vibrational coupling, probably involving significant interactions with phonons associated with the matrix protein. There are, in principle, experimental ways of approaching this question. The exciton model makes the clear prediction that the two exciton states are perpendicular to one another. In the s-to-f model, the s chromophore $(\alpha 84)$ is more nearly parallel than perpendicular to the $f$ chromophore ( $\beta 84)$. Perhaps analysis of the wavelength dependence of time-resolved fluorescence anisotropy will permit this distinction to be made; however, the three-fold symmetry of the $(\alpha \beta)_{3}$-trimers or $(\alpha \beta)_{6}$-hexamers decreases the magnitude of the polarization anisotropy and complicates the analysis. Time-resolved anisotropy relaxation measurements extrapslated back to zero time indicate an initial state that has the maximum polarization expected for isolated chromophores, or for an exciton-split pair where one of the states dominates in the absorption spectrum [23]. By contrast, similar measurements on allophycocyanin show a significantly decreased initial polarization, which was interpreted in terms of exciton splitting for that structurally related molecule. Linear dichroism of oriented phycobiliproteins provides an alternative experimental approach. Results on oriented PC from Nostoc by Juszczac et al. [28] indicated the presence of three resolved transitions in $(\alpha \beta)_{12}$-dodecamers of PC, such that the one at intermediate wavelength is polarized oppositely to that at long wavelength. This is is better agreement with the exciton model than with the s-to-f model; in the latter case the transitions for both the $\alpha 84$ and $\beta 84$ chromophores lie more in the plane perpendicular to the $\mathrm{C}_{3}$ axis than along the axis, at least for $\mathrm{PC}$ from $\boldsymbol{M}$. laminosus or $\boldsymbol{A}$. quadruplicatum. It is encouraging that the LD spectra of Juszczac et al. show that the polarization of the short wavelength $a b$ - 
sorption band, presumably associated with the $\beta 155$ chromophores, is opposite to that of the long wavelength band in PC from Nostoc. The receni linear dichroism study of Schirmer and Vincent [29] on PC crystals from $M$. laminostus support these results. They find a dichroic ratio of 0.2 for the long wavelength absorption, of 1.2 around 605 $\mathrm{nm}$ and between 1.0 and 1.2 below $600 \mathrm{~nm}$ for absorption along (relative to perpendicular to) the crystallographic $c$-axis $\left(\mathrm{C}_{3}\right.$ symmetry axis).

\section{$(\alpha \beta)_{6}$-Hexamer}

The PC from $A$. quadruplicatum crystallizes as a hexameric molecule, which consists of two trimers face-to-face with $C_{2}$ symmetry axes in the plane perpendicular to the $C_{3}$ axis. Calculations using the pure Forster model and the new coordinates for $\boldsymbol{A}$. quadruplicatum $\mathrm{PC}$ indicate very rapid transfer between $\alpha 84$ cliromophores (2.5 ps) and between $\beta 155$ chromophores (5.4 ps) of the two constituent trimers. These exchanges have no effect on the wavelength resolved decay kinetics because they involve excitation transfer between two chromophores of the same spectroscopic class; they should be detectable in studies of timeresolved depolarization, however. The dominant heterochromophoric transfer remains the one between the adjacent $\alpha 84$ and $\beta 84$ chromophores within each trimer. As a conscquence the fastest exponential decay component calculated (Table III) shows little difference between the $(\alpha \beta)_{3}$-trimer and the $(\alpha \beta)_{6}$-hexamer. There should be, however, an increase in the rate of excitation transfer away from the $\beta 155$ chromophores in the $(\alpha \beta)_{6}$-hexamer. For a given $\beta 155$ chromophore there is a new path to an $\alpha 84$ chromophore in the opposite trimer for which the rate constant is about twice as fast as the step from the $\beta 155$ to the $\beta 84$ chromophore of the same $\beta$-subunit. As a consequence, the lifetime of the intermediate fluorescence decay compoient is decreased from 22 ps in the $(\alpha \beta)_{3}$-trimer to $7.5 \mathrm{ps}$ in the $(\alpha \beta)_{6}$-hexamer. The wavelength-resolved fluorescence decay analysis of Holzwarth et al. [22] associated excitation transfer from the shortest wavelength absorbing chromophore ( $\beta 155)$ with a component with a 10 ps lifetime in the $(\alpha \beta)_{6}$-hexamer of PC from Synechococcus 6301. Assuming an arrangement of chromophores similar to that in A. quadruplica- tum, this would support the above interpretation. As with the trimer, however, we cannot properly interpret intermediate decay components observed from the $(\alpha \beta)_{6}$-hexamer.

Calculations of interaction energies between trimers using the alternative exciton model (Table IV) for the $(\alpha \beta)_{6}$-hexamer indicate several that may be detectable, although all are small compared with the component absorption bandwidthe (FWHM $850 \mathrm{~cm}^{-1}$ ) and none is as large as the $\alpha 84 / \beta 84$ interaction already present in the trimers. To explore these contributions further we need a better theoretical understanding of the transitional region where the exciton interactions begin to become prominent in the excitation delocalization process [9].

\section{Acknowledgements}

We wish to thank T. Schirmer and $\mathbf{R}$. Huber (MPI für Biochemie, Martinsried), S. Schneider (Institut für Physikalische Chemie, Technische Universität München, Garching) aủủ A.R. Holzwarth (MPI für Strahlenchemie, Mujlheim) for providing unpublished data and for many helpful discussions. The help of Dr. S. Holbrook (Berkeley) in the preparation of the figures is gratefully acknowledged. This work was supported by the Deutsche Forschungsgemeinschaft (SFB 143 and the CIP botany computer facilities, H.S.), by the Alexander-von-Humboldt Stiftung, Bonn (award to K S.) and by the Office of Energy Research, Office of Basic Energy Sciences, Biological Energy Research Division of the U.S. Department of Energy under Contract DE-AC03-76SF00098.

\section{References}

1 Sauer, K. (1986) in Encyclopedia of Plant Physiology (New Series), Vol. 19. Photosynthesis III (Staehelin, L.A. and Arntzen, C.J., eds.), pp. 85-97, Springer-Verlag, Berlin.

2 Sauer, K. (1975) in Bioenergetics of Photosynthesis (Govindjee, ed.), pp. 115-181, Academic Press, New York.

3 Stachelin, L.A. (1986) in Encyclopedia of Plant Physiology (New Series), Vol. 19, Photosynthesis III (Staehelin, L.A. and Amtzen, C.J., eds.), 1-84, Springer-Verlag, Berlin.

4 Förster, T. (1967) in Comprehensive Biochemistry (Florkin, M. and Stotz, E.H., eds.), Vol. 22, pp. 61-80 Elsevier, Amsterdam.

S Teale, F.W.J. and Dale, R.E. (1970) Biochem. J. 116, 161-169. 
6 Holzwarth, A.R. (1986) in Encyclopedia of Plant Physiology (New Series), Vol. 19, Photosynthesis III (Staehelin, L.A. and Arntzen. C.J., eds.), pp. 299-309, Springer-Verlag, Berlin.

7 Scheer, H. (1986) in Encyclopedia of Plant Physiology (New Series), Vol. 19, Photosynthesis III (Staehelin, L.A. and Arntzen, C.J., eds.), pp. 327-337, Springer-Verlag, Berlin.

8 MacColl, R., Csatorday, K., Berns, D.S. and Traeger, E. (1980) Biochemistry 19, 2817-2820.

9 Kenkre, V.M. and Knox, R.S. (1974) Phys. Rev. Lett. 33, 803-806.

10 Pearlstein, R.M. and Hemenger, R.P. (1978) Proc. Natl. Acad. Sci. USA 75, 4920-4924.

11 Knapp, E.W., Scherer, P.O.J. and Fischer, S.F. (1986) Biochim. Biophys. Acta 852, 295-305.

12 Matthews, B.W. and Fenna, R.E. (1980) Acc. Chem. Res. 13, 309-317.

13 Deisenhofer, J., Epp, O., Miki, K., Huber, R. and Michel, H. (1985) Nature 318, 618-624.

14 Schirmer, T., Bode, W., Huber, R., Sidler, W. and Zuber, H. (1985) J. Mol. Biol. 184, 275-277.

15 Schirmer, T., Huber, R., Schneider, M., Bode, W., Miller, M. and Hackert, M.L. (1986) J. Mol. Biol. 188, 651-676.

16 Sauer, K., Scheer, H. and Sauer, P. (1987) Photochem. Photobiol. 46, 427-440.

17 Schirmer, T., Rode, W. and Huber, R. (1987) J. Mol. Biol. 196, 677-695.
18 Moog, R.S., Kuki, A., Fayer, M.D. and Boxer, S.G. (1984) Biochemistry 23, 1564-1571.

19 Tinoco, I., Jr. (1963) Radiat. Res. 20, 133-139.

20 Ebrey, T.G., Becher, B., Mao, B., Kilbride, P. and Honig, B. (1977) J. Mol. Biol. 12, 377 -397.

21 Hefferle, P., Geiselhart, P., Mindl, T., Schneider, S., John, W. and Scheer, H. (1984) Z. Naturforsch. 39C, 606-616.

22 Holzwarth, A.R., Wendler, J. and Suter, G.W. (1987) Biophys. J. 51, 1-12.

23 Gillbro, T., Sandström, А̊., Sandström, V., Fischer, R. and Scheer, H. (1988) in Photosynthetic Light Harvesting Systems (Scheer, H. and Schneider, S., eds.), pp. 457-468, W. de Gruyter, Berlin.

24 Schneider, S., Geiselhart, P. and Baumann, F. (1988) in Photosynthetic Light Harvesting Systems (Scheer, H. and Schneider, S., eds.), pp. 469-482, W. de Gruyter, Berlin.

25 Wendler, J., John, W., Scheer, H. and Holzwarth, A.R. (1986) Photochem. Photobiol. 44, 79-85.

26 Sandström, A., Gillbro, T., Sundström, V., Fischer, R. and Scheer, H. (1988) Biochim. Biophys. Acta 933, 42-53.

27 Schneider, S., Geiselhart, P., Siebzehnribl, S., Fischer, R. and Scheer, H. (1988) Z. Naturforsch. 43c, 55-62.

28 Juszczac, L., Zilinskas, B.A., Geacintov, N.E. and Breton, J. (1988) in Photosynthetic Light Harvesting Systems (Scheer, H. and Schneider, S., eds.), pp. 281-292, W. de Gruyter, Berlin.

29 Schirmer, T. and Vincent, M.G. (1987) Biochim. Biophys. Acta 893, 379-385. 